\title{
Neurocognitive adaptation and mental health vulnerability following maltreatment: the role of social functioning
}

\author{
McCrory, E.J. ${ }^{1,2}$, Ogle, J.R. ${ }^{1}$, Gerin, M.I. ${ }^{1,2}$, Viding, E. ${ }^{1}$
}

\author{
${ }^{1}$ Division of Psychology and Language Sciences, University College London, London \\ ${ }^{2}$ Anna Freud National Centre for Children and Families, London \\ Corresponding Author: Eamon J. McCrory, Division of Psychology and Language Sciences, University \\ College London, Gower Street, London WC1 6BT, UK; Email: e.mccrory@ucl.ac.uk
}

\begin{abstract}
Childhood maltreatment is associated with a life-time increase in risk of mental health disorder. We propose that such vulnerability may stem in large part from altered patterns of social functioning. Here we highlight key findings from the psychological and epidemiological literature indicating that early maltreatment experience compromises social functioning and attenuates social support in ways that increase mental health vulnerability. We then review the extant neuroimaging studies of children and adolescents, focusing on three domains implicated in social functioning: threat processing, reward processing and emotion regulation. We discuss how adaptations in these domains may increase latent vulnerability to mental health problems by impacting on social functioning via increased stress susceptibility as well as increased stress generation. Finally, we explore how computational psychiatry approaches, alongside systematically reported measures of social functioning, can complement studies of neural function in the creation of a mechanistic framework aimed at informing approaches to prevention and intervention.
\end{abstract}

Keywords: maltreatment, brain, psychiatric disorder, social functioning, computational psychiatry 


\section{Introduction}

The experience of maltreatment in childhood has profound and enduring consequences for mental health. Individuals with histories of maltreatment have a significantly increased risk of concurrent and future internalizing and externalizing psychiatric disorders (Gilbert et al., 2009). When mental health problems do arise, they tend to be associated with an earlier onset of symptoms, greater comorbidity, and poorer responsiveness to traditional interventions (Agnew-Blais et al., 2016; Nanni et al., 2012). Over the last decade, neuroimaging studies have begun to document alterations in neuro-cognitive functioning associated with maltreatment exposure across a number of domains (Gerin et al., 2017). The theory of latent vulnerability postulates that these alterations can be understood in part as adaptations to early adverse or neglectful environments in line with the notion of experiential canalization; the idea that abilities are shaped by the interaction of our biology and experience (Blair \& Raver, 2012; McCrory \& Viding, 2015). While such adaptations are thought to confer short-term functional advantages in atypical early environments, they are believed to contribute to long term mental health vulnerability. In the current paper, we propose that this vulnerability can in large part be understood in the context of altered social functioning. However, it is highly unlikely that such vulnerability only emerges via a socially mediated pathway. Several other pathways are likely to mediate the association between maltreatment exposure, altered neurocognitive functioning and increased psychiatric risk. Domain specific deficits may exert non-social effects that increase psychiatric vulnerability; for example, altered memory processing may increase one's propensity for rumination, contributing to increased risk of depression (Hoffmann et al., 2018; McCrory, Puetz, et al., 2017). Equally, domain general deficits in cognitive processing (following neglect for example) may compromise functioning in ways that also increase psychiatric vulnerability 
(McLaughlin, Sheridan, \& Lambert, 2014). Here, however, we primarily focus on the potential contribution of a socially-mediated pathway to psychiatric vulnerability.

First, we provide a concise overview of the evidence that maltreatment is associated with a deleterious and enduring impact on social functioning, and that maladaptive social functioning is in turn related to poor mental health outcomes. We then consider these findings in the context of a theoretical framework which describes how observable neurocognitive alterations following exposure to early adverse environments may increase vulnerability to poor mental health. Here we outline putative direct and indirect latent vulnerability pathways that we propose are to a large degree socially mediated. Third, we review findings from extant functional magnetic resonance imaging (fMRI) studies of children and adolescents in relation to threat processing, reward processing and emotion regulation. These neurocognitive domains are highly relevant to social functioning and are implicated in a range of mental health disorders. Finally, we consider the significant work that still needs to be conducted to refine our mechanistic understanding of the relationship between childhood maltreatment, social functioning and mental health. In particular, we highlight the potential contribution of approaches derived from computational psychiatry.

\section{Evidence of maladaptive social functioning following childhood maltreatment}

Social functioning - defined broadly as an individual's ability to perform and fulfil normal social roles (Hirschfeld et al., 2000), is arguably both an important mediator in the development of psychopathology following childhood maltreatment as well as an important developmental outcome in its own right. How maltreatment impacts social functioning has been investigated in a broad range of cross-sectional and longitudinal studies. One line of research has investigated how social competence is perceived by others. In a large national cohort of kindergarten children $(\mathrm{N}=69,116)$, associations were observed between exposure 
to any one type maltreatment and an individual's odds of being ranked in the bottom $10 \%$ of the cohort for teacher-rated social competency $(\mathrm{OR}=2.7 ; \mathrm{CI}=2.3-3.1)$, even after adjusting for a range of potential confounds such as age, sex, socio-economic status and parental schizophrenia (Matheson et al., 2017). This effect was even more pronounced for those who had experienced two or more subtypes of maltreatment $(\mathrm{OR}=5 ; \mathrm{CI}=4-6.4)$. In a study of young adults $(\mathrm{N}=435)$, harsher peer first impressions were found to be formed of those who had witnessed domestic violence in childhood (King 2016).

Compelling evidence that the impact of childhood maltreatment (and adversity more generally) has an enduring effect on social competence comes from recent longitudinal studies that have looked at social functioning across several periods with respect to same individuals. For example, Raby and colleagues used data from the Minnesota Longitudinal Study of Risk and Adaptation (MLSRA) $(\mathrm{N}=267)$ to evaluate whether abuse and neglect during the first five years of life had long term consequences for social competence. Teacher reported competence with peers was measured up to age 16 , followed by competence at forming and maintaining high-quality romantic relationships in adulthood (Raby et al., 2018). Maltreatment was found to be directly associated with lower levels of social competence at each developmental stage (Grades 1-6 and ages 16, 23 and 32), which remained the case even after accounting for the stability of social adjustment across development. Similarly, Flynn et al. (2014) employed a three-wave longitudinal design $(\mathrm{N}=635)$ to test the effects of childhood maltreatment on low self-worth, low quality relationships, and both internalizing and externalizing symptoms across early-mid and mid-late adolescence. Not only did this design allow researchers to study whether vulnerabilities associated with maltreatment in early-mid adolescence remained stable across development, they were also able to test for significant cross-lagged effects whereby a parameter in the second wave shows independent associations with the remaining parameters of interest in the third wave (Kearney, 2017). 
Again, difficulties in these domains endured across time points, with independent associations observed between low maternal relationship quality in wave 2 and both internalizing symptoms and self-esteem in wave 3.

A second line of research has explored functioning within adult romantic relationships. Drawing upon a subsample of participants from the MLSRA ( $=179)$, and using semi-structured interviews and videotaped interactions between study participants and their partners, Labella and colleagues found that childhood abuse and neglect predicted poorer romantic competence (Labella et al. 2018). Kapeleris and Paivio (2011) also investigated romantic competence in a sample of 187 undergraduates. Here, participants with a more severe history of emotional abuse and neglect displayed relative difficulties identifying and expressing emotions (characteristic of alexithymia) and emotional dysregulation and inhibition (characteristic of maladaptive emotional processing), with these emotional competence measures fully mediating the association between emotional abuse and neglect and adult attachment styles in romantic relationships.

Unsurprisingly, these findings indicating poorer social competence are complemented by evidence that maltreatment experience is also associated with increased likelihood of rejection and victimization. In one study, Kim and Cicchetti (2010) asked 421 low SES children attending a summer camp to nominate which peer they liked best and least overall. In line with several earlier results (Bolger et al., 1998; Dodge et al., 1994), emotional maltreatment, physical abuse, and sexual abuse were all predictive of higher peer rejection (being rated as 'least liked'). Moreover, there is now convincing data derived from a longitudinal designs that childhood maltreatment is causally associated with an increased risk of being bullied ('bullying victimization'; Benedini et al. 2016). Analogous patterns of rejection and revictimization have also been reported in adulthood. In a prospective study of adults $(\mathrm{N}=892)$, Widom et al. (2014) found that maltreatment history was associated with an 
increased likelihood of both experiencing and perpetrating intimate partner violence; a result that has since been repeated in a study of over thirty thousand adults who provided retrospective reports of maltreatment history (Afifi et al., 2017).

In addition to evidence of poorer social competence and increased risk of rejection and victimization, research has also suggested that individuals who have experienced childhood maltreatment benefit from weaker social support. Using their prospective sample, Sperry and Widom (2013) found a negative association between documented maltreatment history and self-reported social support in adulthood, thirty years later. This is significant in the context of mental health vulnerability as social support is one factor that is thought to buffer against stressful life events that potentiate risk of developing psychiatric disorders (Harkness et al., 2008; Uhrlass \& Gibb, 2007). Indeed, reduced social support has been directly associated with an increased risk of anxiety and depression (Sperry \& Widom, 2013; van Harmelen, 2016). Prospective cohort studies incorporating several waves of longitudinal data from childhood through to early adulthood have also found that differences in perceived social isolation partially mediate the observed associations between maltreatment and mental health symptoms in later life (Alto et al., 2018; Sheikh, 2017).

While there is a general consensus regarding the significant impact of maltreatment on social functioning and subsequent mental health outcomes, it is somewhat surprising how little we know about the neurocognitive mechanisms that may underpin this association. Yet a growing body of work now demonstrates that maltreatment is associated with altered functioning across a range of neurocognitive domains likely to be directly implicated in key aspects of social functioning.

3. The theory of latent vulnerability: Neurocognitive adaptation within the context of social functioning and mental health 
Neuroimaging studies have provided evidence that maltreatment in childhood results in measurable alterations to a number of neurocognitive systems (Teicher et al., 2016; McCrory et al., 2017). However, how these alterations impact social functioning in ways that may increase risk of future mental health problems has been accorded little explicit focus. We have postulated in our theory of latent vulnerability that such alterations can be viewed as representing in part a functional and adaptive response to early neglectful and/or abusive environments (McCrory \& Viding, 2015). However, such adaptations are equally thought to incur a longer-term cost, as they may mean that the individual is poorly optimized to negotiate the demands of other, more normative environments. Here we consider how these costs may have a particular impact on social functioning and social decision-making. While it is of course the case that patterns of adaptation arise at multiple levels (see Cicchetti, 2016), we focus on neurocognitive functioning as the level of investigation most likely to have immediate translational relevance.

We have previously suggested that these neurocognitive changes can confer latent vulnerability either directly or indirectly (McCrory et al., 2017). Direct effects can be understood as the way in which maltreatment-related neurocognitive changes alter how an individual perceives, processes and responds to the social world around them. For example, altered emotion regulation may have a direct effect on psychological functioning, increasing the degree to which new stressors or indeed everyday challenges burden and tax an individual (Tottenham \& Gabard-Durnam, 2017); this can be understood as an increase in 'stress susceptibility'. Equally, neurocognitive changes may alter how an individual influences their own social experience. Direct effects here capture how individuals may act in ways that precipitate the likelihood of stressor events occurring; this can be considered a form of 'stress generation'. By contrast, indirect effects refer to how maltreatment-related neurocognitive changes influence the way that an individual cumulatively shapes their own social ecology 
over time. As we have already noted, childhood maltreatment history is associated with an increased likelihood of reduced social support in adulthood, even decades after abuse is experienced (Sperry \& Widom, 2013). Such reduced social support may make individuals more vulnerable to stressors when they occur: this is another example of increased stress susceptibility. Equally, there may be an increase in stress generation through a greater likelihood of affiliation with individuals who are violent or abusive.

While the extant neuroimaging research has documented alterations associated with maltreatment across a range of neurocognitive systems that are likely to be crucially involved in social functioning, the precise ways in which these alterations increase latent vulnerability is unclear. A mechanistic understanding of this relationship is essential in order to inform therapeutic strategies that could target distinct cognitive processes that would shift a trajectory of maladaptive social transactions across the life course. As shown below, the paradigms that have been used to date typically evoke single responses that do not involve dyadic or iterative social interactions. This makes it difficult to directly test how altered neurocognitive functioning may compromise social competence and social decision making.

\section{Functional magnetic resonance imaging (fMRI) studies of childhood maltreatment}

In light of our interest in underlying neurocognitive mechanisms, we primarily focus on functional magnetic imaging (fMRI) studies of children and adolescents. As such, a systematic consideration of studies of brain structure and those using animal models is beyond the scope of this review. Interested readers are referred to other excellent reviews of these topics (see Teicher \& Samson, 2016 and McLaughlin et al., 2014 respectively). Given the policy and clinical focus on the specific subgroup of children who are exposed to childhood maltreatment (including physical, sexual and emotional abuse and neglect, as well as exposure to intimate partner violence), we prioritize studies that have investigated these 
populations. Given the small number of extant functional imaging studies in the field of childhood maltreatment, we also include studies of children who have experienced severe neglect as a result of early institutionalization. Other forms of childhood adversity or stress (e.g. death of a close family member, poverty, witnessing community violence etc.) are also important for mental health functioning and neurocognitive development, but are beyond the scope of the current review. To date, extant studies have demonstrated altered functioning in three neurocognitive domains implicated in social processing: threat processing, reward processing and emotion regulation. We first review the evidence of altered functioning in these domains among individuals exposed to maltreatment (or institutionalization) in childhood and adolescence, and then turn to consider how these alterations may be relevant to initiating and sustaining impaired social functioning in subsequent normative environments.

\section{i. $\quad$ Threat processing}

Threat processing is a global term referring to several interactive neural mechanisms that underpin behavioral responses to perceived dangers in order to promote survival (LeDoux, 2003). It encompasses information processing biases that enable the rapid identification of environmental threats; learning mechanisms that facilitate the acquisition or extinction of fear responses; attention mechanisms that determine the difficulty one has in disengaging from threatening stimuli; and emotional responses to potential or actual threats (McLaughlin \& Lambert, 2017).

Within the central nervous system, an integrated network comprised of several limbic regions including the amygdala, anterior cingulate cortex (ACC) and hippocampus, as well as cortical regions including the insula and prefrontal cortex (PFC), plays a key role in fear conditioning, stress response and salience detection (Shin \& Liberzon, 2010). Within this network, research has focused on the role of the amygdala - a limbic region that develops 
rapidly during post-natal life with a sensitive period for environmental modification from late infancy to childhood. Studies of rodents and humans (Gee et al., 2014) suggest that over the course of early development, typical patterns of parental caregiving serve to phasically dampen amygdala reactivity to threatening and ambiguous environmental stimuli, leading to the suppression of stress-induced corticosterone release and stimulus response behaviors biased toward approach rather than avoidance, even with respect to aversive stimuli (Callaghan \& Tottenham, 2016a; Moriceau \& Sullivan, 2006; Tottenham, 2018). Parental absence, by contrast, is thought to impoverish the development of functional and structural connections between the amygdala and prefrontal regulatory regions. This may be in turn reflected at the cognitive level by representations of the external world that are subjectively more threatening (Callaghan \& Tottenham, 2016b). These alterations may be adaptive in early environments which are indeed characterized by elevated threat, but are less optimized for more normative social contexts.

Early human studies employing fMRI focused on children with experience of institutional neglect. Among a small sample of post-institutionalized (PI) children ( $\mathrm{N}=$ 11), Maheu et al. (2010) observed greater neural activity in the left amygdala and left anterior hippocampus when viewing pictures of fearful faces compared to a well-matched sample of non-deprived children $(\mathrm{N}=19)$. Moreover, amygdala responses were negatively correlated with time spent in the child's adoptive home, implying a dose-dependent relationship between threat response and early adversity exposure. Using an emotional face-processing paradigm, Tottenham et al. (2011) found that PI children ( $\mathrm{N}=22)$ displayed elevated amygdala activity to threating and distracting stimuli compared to children reared with their biological families $(\mathrm{N}=22)$. More recently, Silvers et al. (2017) reported in a larger sample of PI children and adolescents $(\mathrm{N}=34)$ that inter-individual variability in amygdala responses to threatening faces was linked to higher parent-reported 
anxiety symptoms among PI youths, but not among comparison youths. This suggests that amygdala-supported attention to threat could be an endophenotype for anxiety among PI individuals. However, the cross-sectional design employed limits any causal inferences about the development of such disorders, with larger samples matched on IQ, socioeconomic status (SES) and pubertal status also needed to reduce the range of potential confounds that could be driving the observed group differences. This is particularly important given evidence on the developmental trajectory of amygdala reactivity among neurotypical groups, with adolescents showing exaggerated activity relative to both children and adults (Hare et al., 2008).

Studies from our own research group have focused on children with documented histories of maltreatment exposure recruited from social services departments in the United Kingdom. Matching children with documented maltreatment experiences $(\mathrm{N}=20)$ with control participants of comparable SES, pubertal stage, IQ and age, we found increased activation of the amygdala as well as the anterior insula when viewing angry relative to neutral faces (E. J. McCrory et al., 2011). The anterior insula has been implicated in the processing of emotionally salient environmental information (Shankman et al., 2014), and is thought to act as a bridge between affective and cognitive processing regions (Berntson et al., 2011). In a subsequent study, we assessed neural response to preattentively presented angry faces which appeared for only $17 \mathrm{~ms}$ and backward masked, such that participants were not consciously aware of having viewed a face during the paradigm. A group of children with documented maltreatment experiences were recruited $(\mathrm{N}=18)$ and a group of well-matched control children (McCrory et al., 2013). Again, children exposed to maltreatment showed a greater amygdala response to angry versus to neutral faces, suggesting that threat response at the neural level does not result from conscious regulatory control. Importantly, this study also found that children with earlier onset of maltreatment 
and longer durations of abuse displayed relatively higher levels of amygdala reactivity, suggesting a role of both developmental timing and the chronicity of exposure. Studies of adults with a history of childhood maltreatment indicate that abuse and neglect can have a long-lasting impact on the threat-processing system (Hein \& Monk, 2017; Klumpers et al. 2017). Moreover, studies of both animals (Vyas et al. 2004) and humans (Cohen et al., 2013; Tottenham et al., 2009) suggest that structural and functional amygdala differences may not be fully reversed after the termination of stress.

How might altered threat processing compromise social functioning in normative environments? The amygdala plays a key role in identifying salient events in the environment and encoding relevant affective information. Alterations in amygdala functioning and reduced prefrontal control are likely therefore to contribute to problematic interpersonal interactions. The ways in which this may occur are not clear. One possibility is that heightened amygdala reactivity may increase hypervigilance to threat (including non-relevant information), leading to an over-attribution of threat cues (Lee \& Hoaken, 2007). Such heightened amygdala reactivity (in the context of weaker prefrontal regulation) may lead to higher levels of threat reactive aggression (Chen et al. 2012), trait anger and conflictual interactions that in turn may either increase the likelihood of stress generation or reduce the likelihood of an individual being able to elicit and sustain positive relationships as shown by psychological research among maltreated children (Hecht et al., 2014). In a recent imaging study of young adults aged 18-22, among participants with higher CTQ scores, low levels of self-reported trait anger (the dispositional tendency to experience a wide range of situations as annoying or frustrating) was found to be correlated with both lower threat-related amygdala activity and higher executive control-related dlPFC activity (Kim et al., 2018; McCrory, 2018). However, whether these domain-specific neural variations actually mediate 
meaningful differences in measures of real-world social functioning such as social network size or the quality of close personal relationships was not tested.

There is also evidence of an avoidance of threatening cues, that may reflect a form of dissociation accompanied by amygdala hypoactivation (Puetz et al., 2016). Whilst this form of response may reduce the experience of distress in the short-term, it may impair the development of effective threat detection over the longer term, even placing individuals at increased risk of re-victimization or PTSD symptomatology, again pertinent to the notion of stress generation (DePrince, 2005; Puetz et al., 2016). Over the longer term, it is possible that disruptions in fronto-amygdala circuitry following childhood maltreatment serve to constrain new threat learning and compromise the updating of accurate information about the world as an individual experiences new environments (Moutoussis et al., 2017).

While the ability to categorize and appropriately respond to facial expressions is invaluable for social exchange, few studies to date have investigated alterations in threat processing using naturalistic paradigms that model elements of the recursive social signaling process that characterize real-life interactions. One notable exception is Tottenham et al. (2011), who during scanning and in a separate parent-child live dyadic interaction employed eye tracking to monitor group differences in saccadic movements. Within the group of children who had experienced institutionalization, eye-contact was found to be negatively correlated with amygdala response to faces (both fear and distracters), both during scanning and the dyadic interaction, such that higher amygdala responses to faces were associated with less eye-contact. This study highlights the important association between amygdala activity and social cues that are likely to contribute to moment to moment dyadic signaling.

\section{ii. $\quad$ Reward processing}


Rewards, broadly defined as desirable outcomes that serve to influence behavior (Delgado, 2007), are a central component in driving incentive-based learning, responding optimally to environmental stimuli, and developing goal-directed behaviors. Reward processing has therefore been conceptualized as comprising three distinct processes: 'liking', 'wanting' and 'learning' (Berridge et al., 2009). At the neural level, converging evidence in animals and humans suggests the centrality of a mesocorticolimbic dopaminergic circuit in guiding these processes, including the orbito-frontal cortex (OFC), the basal ganglia (including the striatum), and also other limbic regions, such as the amygdala and hippocampus (Cardinal et al., 2002). Work in rodents has shown that chronic early life stress is associated with alterations in dopaminergic signaling which is associated with both blunted reward-related behaviors, including the development of anhedonia-like symptoms, as well as increased long-term sensitization to the reinforcing properties of drugs of abuse (Fareri \& Tottenham, 2016; Meaney et al., 2002). Human neuroimaging studies among individuals with or at risk of depression, for which anhedonia is a hallmark symptom, have also reported a pattern of reduced striatal activation during reward processing (Stringaris et al., 2015).

To date, most studies of childhood maltreatment exposure have reported neurocognitive alteration in aspects of both anticipation of reward (i.e. 'wanting') as well consummatory/hedonistic processes (i.e. 'liking'). Consistent with animal research, Mehta et al. (2010) found blunted striatal responses to the anticipation of monetary rewards in a small PI sample ( $\mathrm{N}=12)$. Goff and colleagues (2013) also observed blunted striatal response to 'social reward' cues (e.g. happy faces) among a larger group $(\mathrm{N}=38)$ of PI children and adolescents. Similarly, Dillon et al. (2009) found a pattern of reduced neural response to the anticipation of monetary rewards in the left pallidus (a structure of the basal ganglia that integrates reward information and conveys it to motor cortex via the thalamus) among young adults with significant histories of maltreatment $(\mathrm{N}=13)$, suggesting that the neurocognitive 
recalibrations of the reward system following the experience of childhood maltreatment can be long-lasting.

In a large community sample of adolescents with varying degrees of childhood maltreatment $(\mathrm{N}=106)$, Hanson et al. (2015) found that the severity of emotional neglect was associated with reduced development of striatal neural response to the receipt of monetary rewards. The latter was also found to partially mediate the association between a history of neglect and depressive symptomology two years post-baseline. Using socialreward cues, Dennison et al. (2016) did not find this effect; however, they reported that higher striatal responses to social rewards at baseline predicted lower symptoms 2 years later. Overall these longitudinal studies suggest that blunted/increased reward-related striatal activation following maltreatment may represent a marker of vulnerability to future psychopathology/resilience.

Recent work has begun to explore reward learning processes following maltreatment exposure and how these impact reinforcement-based decision-making. Gerin et al. (2017), implemented a probabilistic passive-avoidance task during which young people with documented experience of maltreatment $(\mathrm{N}=18)$ and carefully matched peers were required to learn what stimuli were associated with a higher chance of winning or losing points. Behavioral differences were not observed across groups. However, at a neural level, when envisaging the outcomes associated with their actions, a history of childhood maltreatment was associated with reduced engagement of a widespread network (including the OFC and the striatum) commonly associated with the encoding of action-outcome contingencies on the basis of expected rewards. Interestingly, such neural alterations related to higher levels of anxiety and mood symptomatology among individuals with a history of maltreatment. This pattern of reduced neural response was interpreted as reflecting relative impairments in the precision of learnt stimulus-value representations (White et al., 2016), although concurrent 
behavioral findings indicative of such blunted reward learning are required in order to provide further support for this hypothesis. Harms et al. (2017) used a similar instrumental reward task that required participants to first learn positive (reward) and negative (punishment) stimulus response associations, and then update those pairings once contingencies were shifted by the experimenter. Among a sample of 44 adolescents ( 22 with documented histories of physical abuse recorded by Child Protective Services in the United States), both reward learning performance and brain activation during learning were correlated with maltreatment exposure. Individuals with maltreatment experience showed poorer performance in punishment avoidance learning, as well as less cognitive flexibility following contingency reversal. Indeed, group effects on "re-learning" deficits over the reversal phase of the task were stronger than those in the initial acquisition phase, suggesting that the additional cognitive demands led to further relative performance decrements among maltreated adolescents. Maltreatment was also associated with reduced reward-related activation in the striatum and ACC during reversal learning, as well as several attentionrelated regions, including bilateral middle frontal gyri, cuneus and cerebellum. This suggest that maltreatment exposure may foster alterations in general reinforcement-based learning mechanisms, in addition to changes in specific socio-emotional processes.

\section{How might altered reward processing compromise social functioning in normative} environments? Successfully navigating the social environment requires us to learn from the positive and negative signals received from others. We use these signals to make iterative adjustments to our own behavior that maximize both short and long term rewards, subject to the constraint of our material and cognitive resources. Maltreatment exposure may impair an individual's capacity to learn how to optimize returns from a normative social environment, and this is likely to have an impact on their ability to cultivate and maintain healthy social 
relationships. Indeed, in a recent behavioral study (Fries \& Pollak, 2016), early experiences of neglectful caregiving were strongly associated with impairments in learning the association between visual cues and their rewarding or motivating significance, which also correlated with increased indiscriminate social behavior. Similarly, Sheridan et al. (2018) showed that early institutionalization leads to task-based disruptions in associative learning that were correlated with poorer social skills and depression; one possibility is that this may be mediated by impairments in the updating of action-value contingencies for emotional responses in social settings. Of course, such disruptions may also exert an impact on psychiatric vulnerability via non-social pathways, with impairments in reinforcement learning thought to subserve syndromes of motivation such as anhedonia (Husain \& Roiser, 2018), although maltreatment exposure may also blunt hedonic responses associated with social interactions. Indeed, social behavior is controlled by reward and motivation-related processes in the orbito-striatal circuit, the amygdala and insula that strongly resemble the neural reward signals identified in the context of non-social reinforcement learning and decision-making (Ruff \& Fehr, 2014). This does not imply that maltreatment exposure inculcates stable, well-defined preferences that are anti-social. Rather, atypical functioning of the reward circuitry may alter momentary social decision making as a result of diminished social reward expectations in ways that frustrate the formation of stable social support networks. This can be understood as an example of how neurocognitive changes (here in respect of the reward processing system), may confer latent vulnerability in an indirect way: specifically, attenuating the development of protective social support networks.

While reduced reward-related neural activation has been associated with the degree of social anhedonia in major depressive disorder (Kupferberg et al., 2016), it is not yet clear whether reward circuitry alterations following maltreatment exposure are implicated in maladaptive social decision making. One promising avenue of future research will be to 
employ structured social exchange paradigms adapted from behavioral game theory to test the neural antecedents of active interpersonal collaboration among children with experience of maltreatment (for a review of these paradigms see Camerer \& Fehr, 2004). For example, Wills et al. (2018) used a public goods game among a sample of young adults to show that prosocial versus selfish tendencies lead to dissociable sets of strategic behavior for a fixed pattern of neural activation, such that free riders display relatively more OFC activity when behaving selfishly, whereas prosocial individuals show greater OFC activity while cooperating. Activity in neural reward circuitry has also been found to predict increased cooperation while playing a trust game (Hughes et al., 2017), with greater orbito-striatal activity when participants believe they are cooperating with friends as opposed to unknown peers - despite equal economic incentives - leading to more prosocial behavior (Fareri et al., 2015). This may represent a reflexive neural mechanism that serves to reaffirm and maintain social relationships in neurotypical populations. Indeed, recent behavioral findings from a PI youth sample show that trust game cooperation rates are negatively associated with duration of orphanage exposure (Pitula et al., 2017). However, such behavioral findings are likely underpinned by multiple neurocognitive alterations; thus, carefully designed neuroimaging studies will be required in the future to understand the joint and separable effects of maltreatment induced neural adaptations on social decision-making.

\section{iii. Emotion regulation}

Emotion regulation refers to the implementation of conscious (explicit) or unconscious (implicit) goal-directed adjustments to the trajectory of a given emotion, wholly or partially altering the nature, magnitude, and duration of our internal or external emotional responses (Ochsner et al., 2012). Neuroimaging studies have traditionally identified a pattern of increased prefrontal activation and decreased activation of emotion-related regions, most 
commonly the amygdala, to be associated with the down-regulation of negative emotions (Ochsner et al., 2004, 2012). Explicit regulation has been associated with activation in several lateral prefrontal (IPFC) and parietal regions implicated in cognitive processes important for volitional affect modulation such as attention, working memory, performance monitoring and selecting goal-appropriate responses (Etkin et al., 2015). Implicit emotion regulation, which is important for the spontaneous inhibition and extinction of fear (Maren \& Quirk, 2004), typically involves activations in the ventral anterior cingulate cortex (vACC) and ventromedial prefrontal cortex (vmPFC). In terms of the tasks and stimuli that have most often been used to measure the neural correlates of differing regulatory capabilities among maltreated groups, significant overlap exists with our earlier discussion of threat processing. Broadly, what separates the study of these neurocognitive domains is how functional imaging is employed as a method. Studies of threat processing tend to focus on the localization of areas more or less active during a task. By contrast, studies of emotion regulation tend to focus on how neural signals flow between multiple brain areas as captured by functional connectivity (the temporal dependency of activation patterns among different brain regions) (O’Reilly et al. 2012; van den Heuvel and Hulshoff Pol 2010).

Studies of children and adolescents with histories of maltreatment have consistently found evidence of atypical focal neural activity in regulatory regions such as the vACC and the 1PFC (Elsey et al., 2015; Puetz et al., 2014, 2016), as well as alterations in frontoamygdala connectivity during implicit regulation tasks. However, the direction of these findings (i.e. whether focal activations or functional connections appear increased or decreased relative to control samples) has varied considerably between studies. For example, in a typically developing group of adolescents $(\mathrm{N}=31)$, Lee et al. (2015) observed that degree of verbal abuse exposure was related to both amygdala hyperactivity and decreased functional connectivity between the right amygdala and vACC during the implicit processing 
of negative facial expressions, implying impaired regulatory control (Etkin, 2011). Similarly, Marusak et al. (2015) employed an inhibitory control task involving the detection of emotive facial expressions to test vACC-amygdala inhibitory connectivity among trauma exposed youth $(\mathrm{N}=14)$ compared to a well-matched control sample $(\mathrm{N}=16)$. Again, while negative vACC-amygdala connectivity was observed among comparison participants, such activation was absent in trauma-exposed youth. In contrast, using a simpler face processing task with a sample of 41 PI children and adolescents compared with 48 never institutionalized agematched controls, Gee et al. (2013) found that group differences only emerged for a subsample of the total participants. Specifically, comparison children showed a typical immature pattern of positively coupled amygdala-mPFC/vACC connectivity (positively correlated coactivations between the amygdala and frontal regions, possibly indicative of limited inhibitory control), whereas PI children displayed a pattern of increased negative amygdala-mPFC/vACC connectivity (negatively correlated coactivations) more consistent with the typical adolescent phenotype. In addition, despite higher levels of anxiety at the group level, negative amygdala-mPFC/vACC coupling was also associated with reduced anxiety among the PI group. Replicating substantial cross-species evidence, the findings suggested that chronic early life stress leads to the accelerated development of amygdala$\mathrm{mPFC} / \mathrm{vACC}$ circuitry, which may be understood as an ontogenetic adaptation to early adversity (Gee, 2016). However, it remains unclear whether such variations in mediofrontalamygdala connectivity development are protective or problematic for mental health over the life course. Most recently, Hart et al. (2018) tested twenty-three adolescents with documented histories of severe physical abuse using a series of dynamic emotional faces (1-s video clips of actors displaying neutral, fearful, angry, sad or happy expressions), which the participants were instructed to simply identify. Participants with maltreatment experience $(\mathrm{N}=20)$ were compared to a group of healthy controls $(\mathrm{N}=27)$, as well as a psychiatric control group 
matched on current diagnoses $(\mathrm{N}=20)$ to help disentangle putative maltreatment effects from those arising from concurrent psychiatric disorder. Adolescents with histories of maltreatment responded faster when recognizing fearful expressions relative to controls, and also showed diminished functional connectivity between the vmPFC and the insula compared to both controls and those with psychiatric disorders. These findings provide support for the specificity of maltreatment related effects of weakened top-down control of vmPFC over the insula, potentially leading to a fear regulation deficit and increased fear sensitivity independent of concurrent psychiatric disorder

Finally, McLaughlin et al. (2015) have provided evidence of neural alterations involved in explicit emotion regulation following exposure to physical and sexual abuse. Here, participants were instructed to modulate their emotional responses to affective images. Maltreated adolescents $(\mathrm{N}=21)$ exhibited increased neural activity in the PFC and dorsal ACC during effortful regulation of negative stimuli, leading to a reduction in amygdala activation relative to passive viewing. Moreover, previously observed differences in amygdala reactivity between maltreated and non-maltreated participants $(\mathrm{N}=21)$ were extinguished after explicit modulation. This suggests that maltreated youths may have needed to engage affective control regions to a greater degree in order to regulate their emotional responses, although whether amygdala reactivity can be successfully modulated by maltreated participants to the same degree will require tasks that employ a continuum of aversive stimuli in the future.

The discrepancies in extant research findings described are perhaps not surprising given marked differences in the age and maltreatment history of participants, as well as the emotion regulation paradigms implemented. Longitudinal studies employing a variety of tasks requiring both the explicit and implicit modulation of affective responses are required to test associations with maltreatment exposure across various stages of 
development. It should also be noted that because the analyses described invariably measured statistical dependencies of activations across discrete brain regions (i.e.

correlations), it is not possible to make strong inferences about the direction of causality from these designs alone (Kahan \& Foltynie, 2013). Methods such as dynamic causal modelling (DCM; Stephan et al., 2010) have been developed to probe effective connectivity, defined as the directed influence that one brain region exerts on another (Friston, 2009), which could be used to formally model the direction of task-based functional activity between specific neural nodes in future research.

\section{How might altered emotion regulation compromise social functioning in normative} environments? Meeting the situational demands of any social encounter requires us to prospectively match our emotion-derived behaviors with those that will confer either maximal benefits or minimize costs to ourselves, given our expectations of others. Impaired regulatory capabilities, mediated by decreased functional connectivity in fronto-amygdala circuitry, may increase the impact of everyday social stressors, reinforcing an individual's stress susceptibility. In addition, poorer emotion regulation might be viewed as one mechanism by which suboptimal responses could frustrate social effectiveness following maltreatment. In a recent study of 500 college students reporting varying degrees of childhood emotional abuse and neglect, Berzenski (2018) found that self-reported affect regulation deficits had a significant indirect effect on increased psychopathology and increased problems in social relationships. Similarly, in a cross-sectional study of adolescents ( $\mathrm{N}=1,139)$, Fritz et al. (2018) found that while emotional expression was positively associated with friendship support (factors shown by the authors to be positively associated with resilience; Fritz, Graaff, et al., 2018), for those with a history of childhood adversity this relationship became negative, implying that low emotional suppression may drive friendship withdrawal. Again, this is an example where altered neurocognitive functioning can be 
considered as having an 'indirect effect' on increasing latent vulnerability by reducing the likelihood that an individual will elicit and sustain protective social relationships that would be expected to buffer the experience of future stressor events.

However, it is important to note that there currently is little direct evidence associating altered neural functioning in regulatory regions with impaired social decisionmaking following maltreatment exposure. A recent study of 79 young children (Park et al., 2018) did find that greater exposure to stressful life events was significantly correlated with weaker functional connectivity between the amygdala and mPFC at rest, which also predicted increased aggressive behavior as reported by parents. However, task-based evidence pertaining to maltreated populations is currently lacking. Nonetheless, it is plausible to assume that less effective emotion regulation will result in stressor experiences exacting a greater toll on physical and mental health (Beauchaine et al. 2011). Indeed, dysregulation of the hypothalamic-pituitary-adrenal axis in response to social stressors has previously been implicated in the increased risk of psychopathology following maltreatment (Bunea et al., 2017).

\section{iv. Discussion}

We have considered how specific neurocognitive domains appear to be calibrated by early exposure to maltreatment and have speculated on what this may mean for social functioning as both a mediating and moderating factor in the later expression of mental illness. Although research pertaining to human brain structure and research in animals was not reviewed here in depth owing to space constraints, findings from these related areas are likely to also have important implications for our understanding of impaired social functioning following early life maltreatment (Hanson et al., 2010; McLaughlin et al., 2014; 
Teicher \& Samson, 2016). Moreover, among the functional studies that were reviewed, there are several limitations that characterize our current understanding.

First, we need to better understand the ways in which alterations among a range of discrete neurocognitive systems may work in concert to potentiate socially deleterious functioning. For example, recent theoretical accounts of emotion regulation (Etkin et al., 2015) have conceived of regulatory actions as the final output of a complex set of perception and valuation processes involving judgments of whether the emotion experienced best serves an individual's current goals, which themselves are a likely function of momentary affect (Huys \& Renz, 2017). How a stimulus is perceived (for example, as more or less threatening) and whether the innate and learnt values of all possible regulatory actions are successfully updated following experience (i.e. value-based learning) will exert significant influence over how emotions are finally regulated. The example of prosocial behavior serves to illustrate this point. While a prosocial behavior such as sharing has been shown to demand significant regulatory activity in the dorsolateral PFC (dlPFC) (Steinbeis, 2018; Ruff et al., 2013), heightened threat reactivity may restrain this capacity. For example, incidental aversive threats operating in the background of a social exchange paradigm (i.e. threats that are unrelated to the incentive structure of the interaction) have been shown to lead to the suppression of neural activity in the dlPFC and a reduced likelihood of cooperation (Engelmann et al., 2018).

Second, it is important to emphasize that extant research has focused only on a limited set of neurocognitive mechanisms; it is likely that adaptations in other cognitive domains with more explicit social functions will be important in contributing to an increased risk of psychopathology. For example, psychological research over recent decades has shown a meaningful association between maltreatment and poorer performance on various indices of social emotional understanding (Luke \& Banerjee, 2013). Impaired social understanding in 
turn is thought to result in a diminished ability to respond appropriately when others express their emotions, increasing the risk social exclusion (Jaffee, 2017). However, neuroimaging studies have yet to systematically explore the neurocognitive processes implicated in social understanding.

Third, it is important to acknowledge that the extant functional neuroimaging literature has yet to shed clear light on the potential differential impact of different forms of maltreatment experience. In light of the fact that polyvictimisation typically characterizes the experience of individuals exposed to maltreatment (Berzenski \& Yates, 2011; McLaughlin et al., 2018), it is very difficult (and often impossible) in experimental studies with small sample sizes to reliably establish whether a particular subtype of maltreatment has a unique effect on functional brain activation (Button et al., 2013; Yarkoni, 2009). In the wider literature, we know that there is limited evidence for specific maltreatment types being associated with specific outcomes: different forms of maltreatment appear to have largely equivalent psychiatric and behavioral effects, including anxiety depression and conduct problems (Cecil et al., 2017; Vachon et al., 2015). This does not necessarily imply, however, that common underlying neurocognitive mechanisms instantiate vulnerability. A recent systematic review by Cassiers and colleagues (2018) including both functional and structural studies provides some evidence for long term differential effects of maltreatment subtypes on the human brain. One broad distinction that has been made is in relation to those forms of abuse associated with direct harm (physical, emotional and sexual abuse) and those associated with deprivation-related experiences (such as neglect). Sheridan and McLaughlin, in an important theoretical contribution, have proposed that the former are likely associated with alterations in neural circuits that underlie emotion and emotional learning, while the latter are associated with broader neurocognitive consequences as a result of impoverished cognitive and sensory stimulation throughout development (McLaughlin et al., 2014; McLaughlin et al., 2016; 
Sheridan \& McLaughlin, 2014). One recent study of young adults provides some support for this view (Puetz et al., in press). Neural reactivity to facial cues signalling threat were compared across individuals retrospectively reporting experience of active abuse versus a propensity score matched group reporting only experience of neglect $(\mathrm{N}=87)$. In line with McLaughlin and Sheridan's proposal, it was found that while childhood abuse was associated with heightened localised threat reactivity in the ventral amygdala, experiences of neglect were associated with heightened reactivity in a distributed cortical fronto-parietal network supporting complex social and cognitive processing as well as in the dorsal amygdala (Puetz et al., in press). Among the studies meeting our selection criteria for review, only two involved a direct comparison (either in primary or secondary analysis) of neural activations for exposed and unexposed individuals to distinct maltreatment subtypes controlling for the effects of other subtypes. Lee and colleagues (2015) isolated the effects of emotional abuse by excluding all participants reporting a history of other forms abuse, while Hanson and colleagues (2015) covaried the influence of other forms of abuse in order to investigate the potential unique effect of emotional neglect. Looking forward, rather than focusing on the particular form of maltreatment experience in isolation, it may be more relevant to consider timing and duration alongside. Functional imaging studies, however, have not typically measured onset and duration of maltreatment experience, despite evidence of distinct regionspecific structural changes associated with maltreatment exposures at different development stages (Teicher \& Samson, 2016). Moreover, a recent community based study has highlighted the importance of going beyond a simple focus on subytype. In a latent class analysis approach with a sample of 674 low-income urban children, Warmingham and colleagues (2018) found that those who had experienced maltreatment fell into one of three groups: those who had experienced chronic, multi subtype maltreatment (57\%); those who had experienced neglect only in a single developmental period (31\%); and those characterized by 
a single subtype of maltreatment occurring in a single developmental period (12\%). This is consistent with the view that a focus on a single maltreatment subtype (other than perhaps neglect) is unlikely to be particularly meaningful in relation to children's experiences in the real world.

Finally, it is important to note that we have not considered the impact of genetic factors related to alterations in neural response in the context of maltreatment. None of the studies we have described incorporated molecular genetic data into statistical models when estimating associations between maltreatment and task-induced neural activity. Such imaging genetics approaches can support more accurate estimation by controlling for genetically determined traits that moderate associations between maltreatment and the independent variables of interest, providing greater precision when attempting to isolate key neurobiological pathways involved in both disease risk and resilience (Munoz et al., 2009). To our knowledge, only one functional study of children and adolescents with maltreatment experience has adopted such a design. In a sample of psychiatrically healthy adolescents $(\mathrm{N}=$ 139), White et al. (2012) observed that degree of emotional neglect was predictive of higher amygdala reactivity during a standard emotional face-processing task, although only among those adolescents with a common variant of the FKBP5 gene linked to HPA axis dysfunction. It must be emphasized that across the wider candidate gene literature, decades of genetic case-control association studies (with and without maltreatment exposure included as a moderating environmental factor) have now yielded many publications with very few consistent replications (McCrory et al., 2011; Arango 2017). For example, in a recent replication study of 37 adult imaging genetics studies of threat-related amygdala activity in response to the same emotional face-processing task, Avinun et al. (2018) found that only three of the 37 genetic variants studied (i.e. single nucleotide polymorphisms, frequently called SNPs - pronounced "snips") replicated in their sample of young adults $(\mathrm{N}=1,117)$. In 
light of these poor rates of replication, findings derived exclusively from small experimental samples should be treated with some caution, given that the effects of single common genetic variants on intermediate neural phenotypes will likely be small.

\section{Looking forward: advancing our understanding of maltreatment, social functioning and resilience}

Developmental neuroscientists have investigated neurocognitive alterations following maltreatment exposure that are believed to be important in understanding long term mental health vulnerability. This research has been particularly important in highlighting that functional neural differences often develop prior to the onset of symptomatology following exposure to maltreatment. These alterations can therefore be considered 'latent' in nature, and as such, give impetus to a preventative approach where help is offered before mental health problems emerge. However, across mental health research (including research into the neurocognitive effects of early maltreatment), functional neuroimaging procedures have yet to have a major translational impact on clinical practice. One explanation for this limited translational success is that to date, most neuroimaging investigations have been essentially descriptive in nature, identifying disorder-relevant circuits. Yet on their own, such neurocognitive evidence fails to provide a mechanistic account of either circuit function or complex behavior (Stephan et al. 2015).

Understanding how neurocognitive alterations impact social functioning, and ultimately mental health outcomes, will require us to precisely define and measure the component processes underlying social behavior. Moreover, we will need to understand these processes in the context of the dynamic interaction between multiple neurocognitive systems. For experimental researchers, developing this understanding will involve two steps. First, formalizing testable mathematical models that specify the interacting components that are 
hypothesized to generate each individual decision over the course of a task. Second, developing sufficiently naturalistic paradigms able to evoke affective responses typical of those elicited during real-life interactions (Moutoussis, Eldar, \& Dolan, 2017). The latter may be achieved through the use of social exchange paradigms adapted from behavioral game theory (Sharp et al, 2012), while the former reflects recent moves within psychiatry to understand the specific computations performed by the brain (Hauser, et al., 2018) and how these computations may be non-optimal in ways relevant to psychiatric functioning (Moutoussis et al., 2015).

The distinct advantage of formalizing computational models in neuroscientific research is that they can help us to test verbal theories of how neurocognitive adaptations shape behavior (van den Bos et al., 2017). Here, for instance, we have speculated that hypersensitive threat processing, instantiated by increased amygdala reactivity, may impair social functioning via (i) an individual's over-attribution of threat signals, leading to (ii) more conflictual social interactions. Latent variables such as an individual's perception of threatening stimuli or their degree of hostile attribution bias often cannot be measured directly, but can instead be extracted from a computational model estimated from participants' behavior over a task, again highlighting the need for experimental paradigms able to capture at least some of the computational richness of interpersonal exchange (King-Casas \& Chiu, 2012; Krakauer et al., 2017; Montague, 2018). These variables (known as model parameters) can, in turn, be regressed against measures of task-based neural activity to determine where such latent variables are computed in the brain (Charpentier \& O'Doherty, 2018). At present, the majority of the computational treatments of psychiatric symptomology pertain to models of aberrant learning and decision-making (Stephan \& Mathys, 2014). For example, a learning model derived from a social feedback task was recently able to account for variability in the degree to which participants updated their self-esteem following positive and negative social 
approval ratings, with vulnerability to feedback from others found to be correlated with insula-vmPFC connectivity during self-esteem updates (Will et al., 2017). With researchers already combining therapies such as CBT with neuroimaging in order to test their efficacy using neuroanatomical markers of change (e.g. Mason et al. 2017), computational modeling of social learning and decision-making after childhood maltreatment exposure may provide important mechanistic insights about specific neural and behavioral markers of vulnerability. However, whether computational modelling will have a tangible impact on approaches to prevention and intervention remains to be seen. (Montague, 2018; Stephan \& Mathys, 2014).

In addition to pursuing greater mechanistic clarity through computational modeling, collecting measures of social functioning in the 'real world' alongside experimental data will be an important step in addressing the predictive validity of task-based neural and behavioral measures. With the noted exception of Tottenham and colleagues (2011), extant research has not systematically reported measures of social functioning outside of the scanner that can be related to task-based neuroimaging data. It will be important for future studies to include both subjective measures pertaining to social processes (e.g. relationship satisfaction or perceived social support), as well as more objective assessments such as third party reports or the relative connectedness of individuals within defined social networks (Luke \& Harris, 2007). Integrating these measures into neuroimaging research, as well as computational models, will be necessary to develop mechanistic accounts of how alterations in neurocognitive functioning relate to resilient outcomes following maltreatment experience. Social inputs at multiple levels of the child's ecology (i.e. parent, peer, school, and community support) have been shown to be pivotal in the probabilistic emergence of resilient outcomes longitudinally via the fostering of social skills (Cicchetti, 2016; Oshri et al., 2017). Interventions targeting social functioning, and social skills in particular have been shown to hold particular promise (Cicchetti, 2016; Oshri et al., 2017). One possibility is that such interventions help children 
'recalibrate' the functioning of distinct neurocognitive domains that have been shaped by aberrant social influences from carers earlier in life. We suggest that combining computational approaches, functional neuroimaging, and the longitudinal study of multidimensional measures of children's interpersonal functioning has the potential to inform therapeutic strategies that could more precisely target distinct cognitive processes. Such targeting of specific cognitive processes increases the likelihood that we can shift patterns of maladaptive social transactions in ways that could promote more resilient outcomes over time.

\section{Conclusions}

We suggest that one key way that neurocognitive alterations following childhood maltreatment exposure impacts mental health risk is via social functioning. Specifically, such alterations may compromise the ability of an individual to negotiate stress (increased stress susceptibility) and elevate the probability of future stressful events occurring (increased stress generation). These influences on an individual's social experience may be understood as arising either in the form of a direct effect (i.e. pertaining to online immediate social experience), or an indirect effect (i.e. pertaining to how an individual cumulatively shapes their social ecology over time). We have highlighted key findings that demonstrate impairments in social functioning and weaker social support in individuals who have experienced maltreatment in childhood. We have also reviewed the extant neuroimaging evidence in children and adolescents in three neurocognitive domains implicated in social functioning: threat processing, reward processing and emotion regulation. We propose that altered functioning of these systems following adaptation to early adverse environments may compromise social functioning in more normative environments in the longer term. However, we are aware that other systems may be 
implicated in instantiating this vulnerability. Recently, for example, we have reported that adolescents who have experienced maltreatment present with significant differences in autobiographical memory functioning, including a pattern of over-general recall that may negatively impact social functioning by making it more difficult to draw on past experiences when negotiating social situations (McCrory et al., 2017). Understanding how alterations in neurocognitive systems impact social functioning represents, in our view, a crucial step in explaining the link between early adverse experience and later psychiatric vulnerability. Such a proposal is informed by the central role that effective social functioning plays in sustaining good mental health - both in helping us successfully negotiate stress and life challenges but also in cultivating and sustaining a network of supportive relationships that help mitigate the impact of stressors when they arise.

Currently, how altered functioning of neurocognitive systems following maltreatment influence social decision-making and functioning in differing contexts and across several developmental stages is poorly understood. The development of a more precise mechanistic understanding of impaired social functioning will have important consequences for both therapeutic and preventative strategies. If we better understand how mental health problems unfold over time through the cumulative impact on social functioning, we will be much better placed to develop novel strategies to support young people who have experienced maltreatment, increasing the likelihood of a positive outcome. Preliminary evidence in the field is promising. For example, DePrince et al. (2015) found that a 12-session group intervention with adolescents with histories of maltreatment, which included teaching mindfulness, problem-solving and accurate threat detection, led to a nearly five-fold decrease in reports of sexual victimization over a subsequent six month period. The active ingredients of this approach and whether they pertain to social functioning remain unclear. We believe that computational models of 
maladaptive social behavior, developed through the use of naturalistic social-exchange paradigms, alongside more systematic measurement of social functioning across domains, has the potential to play an important role in shedding light on which aspects of our interventions drive change, as well helping us better understand and predict individual response variation.

\section{References}

Afifi, T. O., Mota, N., Sareen, J., \& MacMillan, H. L. (2017). The relationships between harsh physical punishment and child maltreatment in childhood and intimate partner violence in adulthood. BMC Public Health, 17, 493. https://doi.org/10.1186/s12889017-4359-8

Agnew-Blais, J. C., Polanczyk, G., Danese, A., Wertz, J., Moffitt, T. E., \& Arseneault, L. (2016). Persistence, Remission and Emergence of ADHD in Young Adulthood: Results from a Longitudinal, Prospective Population-Based Cohort. JAMA Psychiatry, 73(7), 713720. https://doi.org/10.1001/jamapsychiatry.2016.0465

Alto, M., Handley, E., Rogosch, F., Cicchetti, D., \& Toth, S. (2018). Maternal relationship quality and peer social acceptance as mediators between child maltreatment and adolescent depressive symptoms: Gender differences. Journal of Adolescence, 63, 19-28. https://doi.org/10.1016/j.adolescence.2017.12.004

Arango, C. (2017). Candidate gene associations studies in psychiatry: time to move forward. European Archives of Psychiatry and Clinical Neuroscience, 267(1), 1-2. https://doi.org/10.1007/s00406-016-0765-7

Avinun, R., Nevo, A., Knodt, A. R., Elliott, M. L., \& Hariri, A. R. (2018). Replication in Imaging Genetics: The Case of Threat-Related Amygdala Reactivity. Biological Psychiatry, 84(2), 148-159. https://doi.org/10.1016/j.biopsych.2017.11.010 
Beauchaine, T. P., Neuhaus, E., Zalewski, M., Crowell, S. E., \& Potapova, N. (2011). The effects of allostatic load on neural systems subserving motivation, mood regulation, and social affiliation. Development and Psychopathology, 23(4), 975-999. https://doi.org/10.1017/S0954579411000459

Benedini, K. M., Fagan, A. A., \& Gibson, C. L. (2016). The cycle of victimization: The relationship between childhood maltreatment and adolescent peer victimization. Child Abuse \& Neglect, 59, 111-121. https://doi.org/10.1016/j.chiabu.2016.08.003

Berntson, G. G., Norman, G. J., Bechara, A., Tranel, D., Bruss, J., \& Cacioppo, J. T. (2011). The insula, the amygdala and evaluative processes. Psychological Science, 22(1), 80-86. https://doi.org/10.1177/0956797610391097

Berridge, K. C., Robinson, T. E., \& Aldridge, J. W. (2009). Dissecting components of reward: 'liking', 'wanting', and learning. Current Opinion in Pharmacology, 9(1), 65-73. https://doi.org/10.1016/j.coph.2008.12.014

Berzenski, S. R. (2018). Distinct emotion regulation skills explain psychopathology and problems in social relationships following childhood emotional abuse and neglect. Development and Psychopathology, 1-14. https://doi.org/10.1017/S0954579418000020

Berzenski, S. R., \& Yates, T. M. (2011). Classes and Consequences of Multiple Maltreatment: A Person-Centered Analysis. Child Maltreatment, 16(4), 250-261. https://doi.org/10.1177/1077559511428353

Blair, C., \& Raver, C. C. (2012). Child Development in the Context of Adversity. The American Psychologist, 67(4), 309-318. https://doi.org/10.1037/a0027493 
Bolger, K. E., Patterson, C. J., \& Kupersmidt, J. B. (1998). Peer Relationships and Self-Esteem among Children Who Have Been Maltreated. Child Development, 69(4), 1171-1197. https://doi.org/10.1111/j.1467-8624.1998.tb06166.x

Bunea, I. M., Szentágotai-Tătar, A., \& Miu, A. C. (2017). Early-life adversity and cortisol response to social stress: a meta-analysis. Translational Psychiatry, 7(12), 1274. https://doi.org/10.1038/s41398-017-0032-3

Button, K. S., loannidis, J. P. A., Mokrysz, C., Nosek, B. A., Flint, J., Robinson, E. S. J., \& Munafò, M. R. (2013). Power failure: why small sample size undermines the reliability of neuroscience. Nature Reviews. Neuroscience, 14(5), 365-376. https://doi.org/10.1038/nrn3475

Callaghan, B. L., \& Tottenham, N. (2016a). The Neuro-Environmental Loop of Plasticity: A Cross-Species Analysis of Parental Effects on Emotion Circuitry Development Following Typical and Adverse Caregiving. Neuropsychopharmacology, 41(1), 163176. https://doi.org/10.1038/npp.2015.204

Callaghan, B. L., \& Tottenham, N. (2016b). The Stress Acceleration Hypothesis: Effects of early-life adversity on emotion circuits and behavior. Current Opinion in Behavioral Sciences, 7, 76-81. https://doi.org/10.1016/j.cobeha.2015.11.018

Camerer, C. F., \& Fehr, E. (2004). Measuring Social Norms and Preferences Using Experimental Games: A Guide for Social Scientist s. Oxford University Press. Retrieved from http://www.oxfordscholarship.com/view/10.1093/0199262055.001.0001/acprof9780199262052-chapter-3 
Cardinal, R. N., Parkinson, J. A., Hall, J., \& Everitt, B. J. (2002). Emotion and motivation: the role of the amygdala, ventral striatum, and prefrontal cortex. Neuroscience and Biobehavioral Reviews, 26(3), 321-352.

Cassiers, L. L. M., Sabbe, B. G. C., Schmaal, L., Veltman, D. J., Penninx, B. W. J. H., \& Van Den Eede, F. (2018). Structural and Functional Brain Abnormalities Associated With Exposure to Different Childhood Trauma Subtypes: A Systematic Review of Neuroimaging Findings. Frontiers in Psychiatry, 9. https://doi.org/10.3389/fpsyt.2018.00329

Cecil, C. A. M., Viding, E., Fearon, P., Glaser, D., \& McCrory, E. J. (2017). Disentangling the mental health impact of childhood abuse and neglect. Child Abuse \& Neglect, 63(Supplement C), 106-119. https://doi.org/10.1016/j.chiabu.2016.11.024

Charpentier, C. J., \& O'Doherty, J. P. (2018). The application of computational models to social neuroscience: promises and pitfalls. Social Neuroscience, 13(6), 637-647. https://doi.org/10.1080/17470919.2018.1518834

Chen, P., Coccaro, E. F., Lee, R., \& Jacobson, K. C. (2012). Moderating effects of childhood maltreatment on associations between social information processing and adult aggression. Psychological Medicine, 42(6), 1293-1304. https://doi.org/10.1017/S0033291711002212

Cicchetti, D. (2016). Socioemotional, Personality, and Biological Development: Illustrations from a Multilevel Developmental Psychopathology Perspective on Child Maltreatment. Annual Review of Psychology, 67, 187-211. https://doi.org/10.1146/annurev-psych-122414-033259 
Cohen, M. M., Tottenham, N., \& Casey, B. (2013). Translational developmental studies of stress on brain and behavior: Implications for adolescent mental health and illness? Neuroscience, 249, 53-62. https://doi.org/10.1016/j.neuroscience.2013.01.023

Delgado, M. R. (2007). Reward-Related Responses in the Human Striatum. Annals of the New York Academy of Sciences, 1104(1), 70-88. https://doi.org/10.1196/annals.1390.002

Dennison, M. J., Sheridan, M. A., Busso, D. S., Jenness, J. L., Peverill, M., Rosen, M. L., \& McLaughlin, K. A. (2016). Neurobehavioral markers of resilience to depression amongst adolescents exposed to child abuse. Journal of Abnormal Psychology, 125(8), 1201-12012. https://doi.org/10.1037/abn0000215

DePrince, A. P. (2005). Social Cognition and Revictimization Risk. Journal of Trauma \& Dissociation, 6(1), 125-141. https://doi.org/10.1300/J229v06n01_08

DePrince, A. P., Chu, A. T., Labus, J., Shirk, S. R., \& Potter, C. (2015). Testing Two Approaches to Revictimization Prevention Among Adolescent Girls in the Child Welfare System. Journal of Adolescent Health, 56(2), S33-S39. https://doi.org/10.1016/j.jadohealth.2014.06.022

Dillon, D. G., Holmes, A. J., Birk, J. L., Brooks, N., Lyons-Ruth, K., \& Pizzagalli, D. A. (2009). Childhood adversity is associated with left basal ganglia dysfunction during reward anticipation in adulthood. Biological Psychiatry, 66(3), 206-213. https://doi.org/10.1016/j.biopsych.2009.02.019

Dodge, K. A., Pettit, G. S., \& Bates, J. E. (1994). Effects of physical maltreatment on the development of peer relations. Development and Psychopathology, 6(1), 43-55. https://doi.org/10.1017/S0954579400005873 
Elsey, J., Coates, A., Lacadie, C. M., McCrory, E. J., Sinha, R., Mayes, L. C., \& Potenza, M. N. (2015). Childhood trauma and neural responses to personalized stress, favorite-food and neutral-relaxing cues in adolescents. Neuropsychopharmacology, 40(7), 15801589. https://doi.org/10.1038/npp.2015.6

Engelmann, J. B., Meyer, F., Ruff, C. C., \& Fehr, E. (2018). The Neural Circuitry Of EmotionInduced Distortions Of Trust. BioRxiv, 129130. https://doi.org/10.1101/129130

Etkin, A., Büchel, C., \& Gross, J. J. (2015). The neural bases of emotion regulation. Nature Reviews Neuroscience, 16(11), 693-700. https://doi.org/10.1038/nrn4044

Etkin, A., Egner, T., \& Kalisch, R. (2011). Emotional processing in anterior cingulate and medial prefrontal cortex. Trends in Cognitive Sciences, 15(2), 85-93. https://doi.org/10.1016/j.tics.2010.11.004

Fareri, D. S., Chang, L. J., \& Delgado, M. R. (2015). Computational Substrates of Social Value in Interpersonal Collaboration. Journal of Neuroscience, 35(21), 8170-8180. https://doi.org/10.1523/JNEUROSCI.4775-14.2015

Fareri, D. S., \& Tottenham, N. (2016). Effects of early life stress on amygdala and striatal development. Developmental Cognitive Neuroscience, 19, 233-247. https://doi.org/10.1016/j.dcn.2016.04.005

Flynn, M., Cicchetti, D., \& Rogosch, F. (2014). The Prospective Contribution of Childhood Maltreatment to Low Self-Worth, Low Relationship Quality, and Symptomatology Across Adolescence. Developmental Psychology, 50(9), 2165-2175. https://doi.org/10.1037/a0037162

Fries, A. B. W., \& Pollak, S. D. (2016). The role of learning in social development: Illustrations from neglected children. Developmental Science, 20(2), e12431. https://doi.org/10.1111/desc.12431 
Friston, K. (2009). Causal Modelling and Brain Connectivity in Functional Magnetic Resonance Imaging. PLoS Biology, 7(2). https://doi.org/10.1371/journal.pbio.1000033

Fritz, J., Fried, E. I., Goodyer, I. M., Wilkinson, P. O., \& Harmelen, A.-L. V. (2018). A Network Model of Resilience Factors for Adolescents with and without Exposure to Childhood Adversity. Open Science Framework. https://doi.org/10.17605/OSF.IO/HVTNG

Fritz, J., Graaff, D., M, A., Caisley, H., van Harmelen, A.-L., \& Wilkinson, P. O. (2018). A Systematic Review of Amenable Resilience Factors that Moderate and/or Mediate the Relationship between Childhood Adversity and Mental Health in Young People. Frontiers in Psychiatry, 9. https://doi.org/10.3389/fpsyt.2018.00230

Gee, D. G. (2016). Sensitive Periods of Emotion Regulation: Influences of Parental Care on Frontoamygdala Circuitry and Plasticity: Sensitive Periods of Emotion Regulation. New Directions for Child and Adolescent Development, 2016(153), 87-110. https://doi.org/10.1002/cad.20166

Gee, D. G., Gabard-Durnam, L. J., Flannery, J., Goff, B., Humphreys, K. L., \& Telzer, E. H. (2013). Early developmental emergence of human amygdala - prefrontal connectivity after maternal deprivation. Proceedings of the National Academy of Sciences of the United States of America, 110(39), 15638-15643. https://doi.org/10.1073/pnas.1307893110//DCSupplemental.www.pnas.org/cgi/doi/10.1073/pnas.1307893110

Gee, D. G., Gabard-Durnam, L., Telzer, E. H., Humphreys, K. L., Goff, B., Shapiro, M., ... Tottenham, N. (2014). Maternal buffering of human amygdala-prefrontal circuitry during childhood but not adolescence. Psychological Science, 25(11), 2067-2078. https://doi.org/10.1177/0956797614550878 
Gerin, M. I., Puetz, V. B., Blair, R. J. R., White, S., Sethi, A., Hoffmann, F., ... McCrory, E. J. (2017). A neurocomputational investigation of reinforcement-based decision making as a candidate latent vulnerability mechanism in maltreated children. Development and Psychopathology, 29(5), 1689-1705.

https://doi.org/10.1017/S095457941700133X

Gilbert, R., Widom, C. S., Browne, K., Fergusson, D., Webb, E., \& Janson, S. (2009). Burden and consequences of child maltreatment in high-income countries. The Lancet, 373(9657), 68-81. https://doi.org/10.1016/S0140-6736(08)61706-7

Goff, B., Gee, D. G., Telzer, E. H., Humphreys, K. L., Gabard-Durnam, L., Flannery, J., \& Tottenham, N. (2013). Reduced nucleus accumbens reactivity and adolescent depression following early-life stress. Neuroscience, 249, 129-138. https://doi.org/10.1016/j.neuroscience.2012.12.010

Hanson, J. L., Chung, M. K., Avants, B. B., Shirtcliff, E. A., Gee, J. C., Davidson, R. J., \& Pollak, S. D. (2010). Early Stress Is Associated with Alterations in the Orbitofrontal Cortex: A Tensor-Based Morphometry Investigation of Brain Structure and Behavioral Risk. Journal of Neuroscience, 30(22), 7466-7472. https://doi.org/10.1523/JNEUROSCI.0859-10.2010

Hanson, Jamie L, Hariri, A. R., \& Williamson, D. E. (2015). Blunted Ventral Striatum Development in Adolescence Reflects Emotional Neglect and Predicts Depressive Symptoms. Biological Psychiatry, 78(9), 598-605. https://doi.org/10.1016/j.biopsych.2015.05.010

Hare, T. A., Tottenham, N., Galvan, A., Voss, H. U., Glover, G. H., \& Casey, B. J. (2008). Biological substrates of emotional reactivity and regulation in adolescence during an 
emotional go-nogo task. Biological Psychiatry, 63(10), 927-934.

https://doi.org/10.1016/j.biopsych.2008.03.015015

Harkness, K. L., Lumley, M. N., \& Truss, A. E. (2008). Stress generation in adolescent depression: The moderating role of child abuse and neglect. Journal of Abnormal Child Psychology, 36(3), 421-432. https://doi.org/10.1007/s10802-007-9188-2

Harms, M. B., Shannon Bowen, K. E., Hanson, J. L., \& Pollak, S. D. (2017). Instrumental learning and cognitive flexibility processes are impaired in children exposed to early life stress. Developmental Science, n/a-n/a. https://doi.org/10.1111/desc.12596

Hart, H., Lim, L., Mehta, M. A., Simmons, A., Mirza, K. a. H., \& Rubia, K. (2018). Altered fear processing in adolescents with a history of severe childhood maltreatment: an fMRI study. Psychological Medicine, 48(7), 1092-1101. https://doi.org/10.1017/S0033291716003585

Hauser, T. U., Will, G.-J., Dubois, M., \& Dolan, R. J. (2018). Developmental Computational Psychiatry. PsyArXiv. https://doi.org/10.17605/OSF.IO/85PRQ

Hecht, K. F., Cicchetti, D., Rogosch, F. A., \& Crick, N. (2014). Borderline Personality Features in Childhood: The Role of Subtype, Developmental Timing and Chronicity of Child Maltreatment. Development and Psychopathology, 26(3), 805-815. https://doi.org/10.1017/S0954579414000406

Hein, T. C., \& Monk, C. S. (2017). Research Review: Neural response to threat in children, adolescents, and adults after child maltreatment - a quantitative meta-analysis. Journal of Child Psychology and Psychiatry, 58(3), 222-230. https://doi.org/10.1111/jcpp.12651 
Hirschfeld, R. M., Montgomery, S. A., Keller, M. B., Kasper, S., Schatzberg, A. F., Möller, H. J., ... Bourgeois, M. (2000). Social functioning in depression: a review. The Journal of Clinical Psychiatry, 61(4), 268-275.

Hoffmann, F., Viding, E., Puetz, V. B., Gerin, M. I., Sethi, A., Rankin, G., \& McCrory, E. J. (2018). Evidence for Depressogenic Spontaneous Thoughts and Altered Resting-State Connectivity in Adolescents With a Maltreatment History. Journal of the American Academy of Child \& Adolescent Psychiatry, 57(9), 687-695.e4. https://doi.org/10.1016/j.jaac.2018.05.020

Hughes, B. L., Ambady, N., \& Zaki, J. (2017). Trusting outgroup, but not ingroup members, requires control: neural and behavioral evidence. Social Cognitive and Affective Neuroscience, 12(3), 372-381. https://doi.org/10.1093/scan/nsw139

Husain, M., \& Roiser, J. P. (2018). Neuroscience of apathy and anhedonia: a transdiagnostic approach. Nature Reviews Neuroscience, 1. https://doi.org/10.1038/s41583-0180029-9

Huys, Q. J. M., \& Renz, D. (2017). A Formal Valuation Framework for Emotions and Their Control. Biological Psychiatry, 82(6), 413-420. https://doi.org/10.1016/j.biopsych.2017.07.003

Jaffee, S. R. (2017). Child Maltreatment and Risk for Psychopathology in Childhood and Adulthood. Annual Review of Clinical Psychology, 13(1), 525-551. https://doi.org/10.1146/annurev-clinpsy-032816-045005

Kahan, J., \& Foltynie, T. (2013). Understanding DCM: Ten simple rules for the clinician. Neurolmage, 83, 542-549. https://doi.org/10.1016/j.neuroimage.2013.07.008 Kapeleris, A. R., \& Paivio, S. C. (2011). Identity and Emotional Competence as Mediators of the Relation between Childhood Psychological Maltreatment and Adult Love 
Relationships. Journal of Aggression, Maltreatment \& Trauma, 20(6), 617-635. https://doi.org/10.1080/10926771.2011.595764

Kearney, M. W. (2017). Cross-Lagged Panel Analysis. In The SAGE Encyclopedia of Communication Research Methods (Vols. 1-4, pp. 313-314). Thousand Oaks: SAGE Publications, Inc. https://doi.org/10.4135/9781483381411

Kim, J., \& Cicchetti, D. (2010). Longitudinal pathways linking child maltreatment, emotion regulation, peer relations, and psychopathology. Journal of Child Psychology and Psychiatry, 51(6), 706-716. https://doi.org/10.1111/j.1469-7610.2009.02202.x

Kim, M. J., Scult, M. A., Knodt, A. R., Radtke, S. R., d'Arbeloff, T. C., Brigidi, B. D., \& Hariri, A. R. (2018). A Link Between Childhood Adversity and Trait Anger Reflects Relative Activity of the Amygdala and Dorsolateral Prefrontal Cortex. Biological Psychiatry: Cognitive Neuroscience and Neuroimaging, 3(7), 644-649. https://doi.org/10.1016/j.bpsc.2018.03.006

King, A. R. (2016). Peer First Impressions of Childhood Maltreatment Victims. Journal of Aggression, Maltreatment \& Trauma, 25(2), 164-179. https://doi.org/10.1080/10926771.2016.1121188

King-Casas, B., \& Chiu, P. H. (2012). Understanding Interpersonal Function in Psychiatric Illness Through Multiplayer Economic Games. Biological Psychiatry, 72(2), 119-125. https://doi.org/10.1016/j.biopsych.2012.03.033

Klumpers, F., Kroes, M. C. W., Baas, J., \& Fernández, G. (2017). How human amygdala and bed nucleus of the stria terminalis may drive distinct defensive responses. Journal of Neuroscience, 3830-16. https://doi.org/10.1523/JNEUROSCI.3830-16.2017 
Krakauer, J. W., Ghazanfar, A. A., Gomez-Marin, A., Maclver, M. A., \& Poeppel, D. (2017). Neuroscience Needs Behavior: Correcting a Reductionist Bias. Neuron, 93(3), 480490. https://doi.org/10.1016/j.neuron.2016.12.041

Kupferberg, A., Bicks, L., \& Hasler, G. (2016). Social functioning in major depressive disorder. Neuroscience \& Biobehavioral Reviews, 69, 313-332. https://doi.org/10.1016/j.neubiorev.2016.07.002

Labella, M. H., Johnson, W. F., Martin, J., Ruiz, S. K., Shankman, J. L., Englund, M. M., ... Simpson, J. A. (2018). Multiple Dimensions of Childhood Abuse and Neglect Prospectively Predict Poorer Adult Romantic Functioning. Personality and Social Psychology Bulletin, 44(2), 238-251. https://doi.org/10.1177/0146167217736049

LeDoux, J. (2003). The emotional brain, fear, and the amygdala. Cellular and Molecular Neurobiology, 23(4-5), 727-738.

Lee, S. W., Yoo, J. H., Kim, K. W., Lee, J. S., Kim, D., Park, H., ... Jeong, B. (2015). Aberrant function of frontoamygdala circuits in adolescents with previous verbal abuse experiences. Neuropsychologia, 79, 76-85.

https://doi.org/10.1016/j.neuropsychologia.2015.10.029

Lee, V., \& Hoaken, P. N. S. (2007). Cognition, Emotion, and Neurobiological Development: Mediating the Relation Between Maltreatment and Aggression. Child Maltreatment, 12(3), 281-298. https://doi.org/10.1177/1077559507303778

Luke, D. A., \& Harris, J. K. (2007). Network Analysis in Public Health: History, Methods, and Applications. Annual Review of Public Health, 28(1), 69-93. https://doi.org/10.1146/annurev.publhealth.28.021406.144132

Luke, N., \& Banerjee, R. (2013). Differentiated associations between childhood maltreatment experiences and social understanding: A meta-analysis and systematic 
review. Developmental Review, 33(1), 1-28.

https://doi.org/10.1016/j.dr.2012.10.001

Maheu, F. S., Dozier, M., Guyer, A. E., Mandell, D., Peloso, E., Poeth, K., ... Ernst, M. (2010). A preliminary study of medial temporal lobe function in youths with a history of caregiver deprivation and emotional neglect. Cognitive, Affective \& Behavioral Neuroscience, 10(1), 34-49. https://doi.org/10.3758/CABN.10.1.34

Maren, S., \& Quirk, G. J. (2004). Neuronal signalling of fear memory. Nature Reviews Neuroscience, 5(11), 844-852. https://doi.org/10.1038/nrn1535

Marusak, H. A., Martin, K. R., Etkin, A., \& Thomason, M. E. (2015). Childhood Trauma Exposure Disrupts the Automatic Regulation of Emotional Processing. Neuropsychopharmacology, 40(5), 1250-1258. https://doi.org/10.1038/npp.2014.311

Mason, L., Peters, E., Williams, S. C., \& Kumari, V. (2017). Brain connectivity changes occurring following cognitive behavioural therapy for psychosis predict long-term recovery. Translational Psychiatry, 7(1), e1001-e1001. https://doi.org/10.1038/tp.2016.263

Matheson, S. L., Kariuki, M., Green, M. J., Dean, K., Harris, F., Tzoumakis, S., ... Laurens, K. R. (2017). Effects of maltreatment and parental schizophrenia spectrum disorders on early childhood social-emotional functioning: a population record linkage study. Epidemiology and Psychiatric Sciences, 26(6), 612-623. https://doi.org/10.1017/S204579601600055X

McCrory, E., De Brito, S. A., \& Viding, E. (2011). The Impact of Childhood Maltreatment: A Review of Neurobiological and Genetic Factors. Frontiers in Psychiatry, 2. https://doi.org/10.3389/fpsyt.2011.00048 
McCrory, E. J. (2018). Investigating the Neurocognitive Mechanisms That Influence How Mental Health Risk Can Unfold Following Maltreatment. Biological Psychiatry: Cognitive Neuroscience and Neuroimaging, 3(7), 579-580. https://doi.org/10.1016/j.bpsc.2018.05.003

McCrory, E. J., Brito, S. A. D., Kelly, P. A., Bird, G., Sebastian, C. L., Mechelli, A., ... Viding, E. (2013). Amygdala activation in maltreated children during pre-attentive emotional processing. The British Journal of Psychiatry, 202(4), 269-276. https://doi.org/10.1192/bjp.bp.112.116624

McCrory, E. J., De Brito, S. A., Sebastian, C. L., Mechelli, A., Bird, G., Kelly, P. A., \& Viding, E. (2011). Heightened neural reactivity to threat in child victims of family violence. Current Biology, 21(23), R947-R948. https://doi.org/10.1016/j.cub.2011.10.015 McCrory, E. J., Gerin, M. I., \& Viding, E. (2017). Annual Research Review: Childhood maltreatment, latent vulnerability and the shift to preventative psychiatry - the contribution of functional brain imaging. Journal of Child Psychology and Psychiatry, 58(4), 338-357. https://doi.org/10.1111/jcpp.12713

McCrory, E. J., Puetz, V. B., Maguire, E. A., Mechelli, A., Palmer, A., Gerin, M. I., ... Viding, E. (2017). Autobiographical memory: a candidate latent vulnerability mechanism for psychiatric disorder following childhood maltreatment. The British Journal of Psychiatry, 211(4), 216-222. https://doi.org/10.1192/bjp.bp.117.201798

McCrory, E. J., \& Viding, E. (2015). The theory of latent vulnerability: Reconceptualizing the link between childhood maltreatment and psychiatric disorder. Development and Psychopathology, 27(2), 493-505. https://doi.org/10.1017/S0954579415000115

McLaughlin, K. A., Gureje, O., Kawakami, N., Koenen, K. C., \& Kessler, R. C. (2018, August). The Effects of Childhood Adversities. https://doi.org/10.1017/9781107445130.011 
McLaughlin, K. A., \& Lambert, H. K. (2017). Child trauma exposure and psychopathology: mechanisms of risk and resilience. Current Opinion in Psychology, 14, 29-34. https://doi.org/10.1016/j.copsyc.2016.10.004

McLaughlin, K. A., Peverill, M., Gold, A. L., Alves, S., \& Sheridan, M. A. (2015). Child Maltreatment and Neural Systems Underlying Emotion Regulation. Journal of the American Academy of Child and Adolescent Psychiatry, 54(9), 753-762. https://doi.org/10.1016/j.jaac.2015.06.010

McLaughlin, K. A., Sheridan, M. A., \& Lambert, H. K. (2014). Childhood Adversity and Neural Development: Deprivation and Threat as Distinct Dimensions of Early Experience. Neuroscience and Biobehavioral Reviews, 47, 578-591. https://doi.org/10.1016/j.neubiorev.2014.10.012

McLaughlin, K. A., Sheridan, M. A., \& Nelson, C. A. (2016, October). Neglect as a Violation of Species-Expectant Experience: Neurodevelopmental Consequences. Biological Psychiatry, pp. 462-471. https://doi.org/10.1016/j.biopsych.2017.02.1096

Meaney, M. J., Brake, W., \& Gratton, A. (2002). Environmental regulation of the development of mesolimbic dopamine systems: a neurobiological mechanism for vulnerability to drug abuse? Psychoneuroendocrinology, 27(1-2), 127-138.

Mehta, M. A., Gore-Langton, E., Golembo, N., Colvert, E., Williams, S. C. R., \& Sonuga-Barke, E. (2010). Hyporesponsive reward anticipation in the basal ganglia following severe institutional deprivation early in life. Journal of Cognitive Neuroscience, 22(10), 2316-2325. https://doi.org/10.1162/jocn.2009.21394

Montague, P. R. (2018). Chapter 11 - Computational Phenotypes Revealed by Interactive Economic Games. In A. Anticevic \& J. D. Murray (Eds.), Computational Psychiatry (pp. 273-292). Academic Press. https://doi.org/10.1016/B978-0-12-809825-7.00011-0 
Moriceau, S., \& Sullivan, R. M. (2006). Maternal presence serves as a switch between learning fear and attraction in infancy. Nature Neuroscience, 9(8), 1004-1006. https://doi.org/10.1038/nn1733

Moutoussis, M., Story, G. W., \& Dolan, R. J. (2015). The computational psychiatry of reward: broken brains or misguided minds? Frontiers in Psychology, 6, 1445. https://doi.org/10.3389/fpsyg.2015.01445

Moutoussis, Michael, Eldar, E., \& Dolan, R. J. (2017). Building a New Field of Computational Psychiatry. Biological Psychiatry, 82(6), 388-390. https://doi.org/10.1016/j.biopsych.2016.10.007

Moutoussis, Michael, Shahar, N., Hauser, T. U., \& Dolan, R. J. (2017). Computation in Psychotherapy, or How Computational Psychiatry Can Aid Learning-Based Psychological Therapies. Computational Psychiatry, 2, 50-73. https://doi.org/10.1162/CPSY_a_00014

Munoz, K., Hyde, L. W., \& Hariri, A. (2009). Imaging Genetics. Journal of the American Academy of Child and Adolescent Psychiatry, 48(4), 356-361. https://doi.org/10.1097/CHI.0b013e31819aad07

Nanni, V., Uher, R., \& Danese, A. (2012). Childhood maltreatment predicts unfavorable course of illness and treatment outcome in depression: a meta-analysis. The American Journal of Psychiatry, 169(2), 141-151. https://doi.org/10.1176/appi.ajp.2011.11020335

Ochsner, K. N., Ray, R. D., Cooper, J. C., Robertson, E. R., Chopra, S., Gabrieli, J. D. E., \& Gross, J. J. (2004). For better or for worse: neural systems supporting the cognitive down- and up-regulation of negative emotion. Neurolmage, 23(2), 483-499. https://doi.org/10.1016/j.neuroimage.2004.06.030 
Ochsner, K. N., Silvers, J. A., \& Buhle, J. T. (2012). Functional imaging studies of emotion regulation: a synthetic review and evolving model of the cognitive control of emotion. Annals of the New York Academy of Sciences, 1251, E1-24. https://doi.org/10.1111/j.1749-6632.2012.06751.x

O’Reilly, J. X., Woolrich, M. W., Behrens, T. E. J., Smith, S. M., \& Johansen-Berg, H. (2012). Tools of the trade: psychophysiological interactions and functional connectivity. Social Cognitive and Affective Neuroscience, 7(5), 604-609. https://doi.org/10.1093/scan/nss055

Oshri, A., Topple, T. A., \& Carlson, M. W. (2017). Positive Youth Development and Resilience: Growth Patterns of Social Skills Among Youth Investigated for Maltreatment. Child Development, 88(4), 1087-1099. https://doi.org/10.1111/cdev.12865

Park, A. T., Leonard, J. A., Saxler, P. K., Cyr, A. B., Gabrieli, J. D. E., \& Mackey, A. P. (2018). Amygdala-medial prefrontal cortex connectivity relates to stress and mental health in early childhood. Social Cognitive and Affective Neuroscience, 13(4), 430-439. https://doi.org/10.1093/scan/nsy017

Pitula, C. E., Wenner, J. A., Gunnar, M. R., \& Thomas, K. M. (2017). To trust or not to trust: social decision-making in post-institutionalized, internationally adopted youth. Developmental Science, 20(3). https://doi.org/10.1111/desc.12375

Puetz, V. B., Kohn, N., Dahmen, B., Zvyagintsev, M., Schüppen, A., Schultz, R. T., ... Konrad, K. (2014). Neural response to social rejection in children with early separation experiences. Journal of the American Academy of Child and Adolescent Psychiatry, 53(12), 1328-1337.e8. https://doi.org/10.1016/j.jaac.2014.09.004 
Puetz, V. B., Viding, E., Gerin, M. I., Pingault, J.-B., Sethi, A., Knodt, A. R., ... McCrory, E. J. (in press). Investigating patterns of neural response associated with childhood abuse versus childhood neglect. Psychological Medicine.

Puetz, V. B., Viding, E., Palmer, A., Kelly, P. A., Lickley, R., Koutoufa, I., ... McCrory, E. J. (2016). Altered neural response to rejection-related words in children exposed to maltreatment. Journal of Child Psychology and Psychiatry and Allied Disciplines, 57(10), 1165-1173. https://doi.org/10.1111/jcpp.12595

Raby, K. L., Roisman, G. I., Labella, M. H., Martin, J., Fraley, R. C., \& Simpson, J. A. (2018). The Legacy of Early Abuse and Neglect for Social and Academic Competence From Childhood to Adulthood. Child Development, $0(0)$. https://doi.org/10.1111/cdev.13033

Ruff, C. C., Ugazio, G., \& Fehr, E. (2013). Changing Social Norm Compliance with Noninvasive Brain Stimulation. Science, 342(6157), 482-484. https://doi.org/10.1126/science.1241399

Ruff, Christian C., \& Fehr, E. (2014). The neurobiology of rewards and values in social decision making. Nature Reviews Neuroscience, 15(8), 549. https://doi.org/10.1038/nrn3776

Shankman, S. A., Gorka, S. M., Nelson, B. D., Fitzgerald, D. A., Phan, K. L., \& O’Daly, O. (2014). Anterior Insula Responds to Temporally Unpredictable Aversiveness: an fMRI Study. Neuroreport, 25(8), 596-600. https://doi.org/10.1097/WNR.0000000000000144

Sharp, C., Monterosso, J., \& Montague, R. (2012). Neuroeconomics: A bridge for translational research. Biological Psychiatry, 72(2), 87-92. https://doi.org/10.1016/j.biopsych.2012.02.029 
Sheikh, M. A. (2017). Childhood physical maltreatment, perceived social isolation, and internalizing symptoms: a longitudinal, three-wave, population-based study. European Child \& Adolescent Psychiatry, 1-11. https://doi.org/10.1007/s00787-0171090-z

Sheridan, M. a, \& McLaughlin, K. a. (2014). Dimensions of early experience and neural development: deprivation and threat. Trends in Cognitive Sciences, 1-6. https://doi.org/10.1016/j.tics.2014.09.001

Sheridan, M. A., McLaughlin, K. A., Winter, W., Fox, N., Zeanah, C., \& Nelson, C. A. (2018). Early deprivation disruption of associative learning is a developmental pathway to depression and social problems. Nature Communications, 9(1), 2216. https://doi.org/10.1038/s41467-018-04381-8

Shin, L. M., \& Liberzon, I. (2010). The neurocircuitry of fear, stress, and anxiety disorders. Neuropsychopharmacology : Official Publication of the American College of Neuropsychopharmacology, 35(1), 169-191. https://doi.org/10.1038/npp.2009.83

Silvers, J. A., Goff, B., Gabard-Durnam, L. J., Gee, D. G., Fareri, D. S., Caldera, C., \& Tottenham, N. (2017). Vigilance, the Amygdala, and Anxiety in Youths With a History of Institutional Care. Biological Psychiatry: Cognitive Neuroscience and Neuroimaging, 2(6), 493-501. https://doi.org/10.1016/j.bpsc.2017.03.016

Sperry, D. M., \& Widom, C. S. (2013). Child Abuse and Neglect, Social Support, and Psychopathology in Adulthood: A Prospective Investigation. Child Abuse \& Neglect, 37(6), 415-425. https://doi.org/10.1016/j.chiabu.2013.02.006

Steinbeis, N. (2018). Neurocognitive mechanisms of prosociality in childhood. Current Opinion in Psychology, 20, 30-34. https://doi.org/10.1016/j.copsyc.2017.08.012 
Stephan, K. E., Penny, W. D., Moran, R. J., den Ouden, H. E. M., Daunizeau, J., \& Friston, K. J. (2010). Ten simple rules for dynamic causal modeling. Neurolmage, 49(4), 30993109. https://doi.org/10.1016/j.neuroimage.2009.11.015

Stephan, Klaas Enno, \& Mathys, C. (2014). Computational approaches to psychiatry. Current Opinion in Neurobiology, 25, 85-92. https://doi.org/10.1016/j.conb.2013.12.007 Stephan, Klaas E., Iglesias, S., Heinzle, J., \& Diaconescu, A. O. (2015). Translational Perspectives for Computational Neuroimaging. Neuron, 87(4), 716-732. https://doi.org/10.1016/j.neuron.2015.07.008

Stringaris, A., Vidal-Ribas Belil, P., Artiges, E., Lemaitre, H., Gollier-Briant, F., Wolke, S., ... Paillère-Martinot, M.-L. (2015). The Brain's Response to Reward Anticipation and Depression in Adolescence: Dimensionality, Specificity, and Longitudinal Predictions in a Community-Based Sample. American Journal of Psychiatry, 172(12), 1215-1223. https://doi.org/10.1176/appi.ajp.2015.14101298

Teicher, M. H., \& Samson, J. A. (2016). Annual Research Review: Enduring neurobiological effects of childhood abuse and neglect. Journal of Child Psychology and Psychiatry, 57(3), 241-266. https://doi.org/10.1111/jcpp.12507

Teicher, M., Samson, J., Anderson, C., \& Ohashi, K. (2016). The effects of childhood maltreatment on brain structure, function and connectivity. Nature Reviews Neuroscience, 17, 652-666. https://doi.org/10.1038/nrn.2016.111

Tottenham, N., Hare, T. A., Millner, A., Gilhooly, T., Zevin, J. D., \& Casey, B. J. (2011). Elevated amygdala response to faces following early deprivation. Developmental Science, 14(2), 190-204. https://doi.org/10.1111/j.1467-7687.2010.00971.x 
Tottenham, Nim. (2018). The Fundamental Role of Early Environments to Developing an Emotionally Healthy Brain. Policy Insights from the Behavioral and Brain Sciences, 5(1), 98-103. https://doi.org/10.1177/2372732217745098

Tottenham, Nim, \& Gabard-Durnam, L. J. (2017). The developing amygdala: a student of the world and a teacher of the cortex. Current Opinion in Psychology, 17, 55-60. https://doi.org/10.1016/j.copsyc.2017.06.012

Tottenham, Nim, Hare, T. A., Quinn, B. T., McCarry, T. W., Nurse, M., Gilhooly, T., ... Casey, B. J. (2009). Prolonged institutional rearing is associated with atypically large amygdala volume and difficulties in emotion regulation. Developmental Science, 13(1), 46-61. https://doi.org/10.1111/j.1467-7687.2009.00852.x

Uhrlass, D. J., \& Gibb, B. E. (2007). Childhood Emotional Maltreatment and The Stress Generation Model of Depression. Journal of Social and Clinical Psychology, 26(1), 119-130. https://doi.org/10.1521/jscp.2007.26.1.119

Vachon, D. D., Krueger, R. F., Rogosch, F. A., \& Cicchetti, D. (2015). Assessment of the Harmful Psychiatric and Behavioral Effects of Different Forms of Child Maltreatment. JAMA Psychiatry, 72(11), 1135-1142. https://doi.org/10.1001/jamapsychiatry.2015.1792

van den Bos, W., Bruckner, R., Nassar, M. R., Mata, R., \& Eppinger, B. (2017). Computational neuroscience across the lifespan: Promises and pitfalls. Developmental Cognitive Neuroscience. https://doi.org/10.1016/j.dcn.2017.09.008

van den Heuvel, M. P., \& Hulshoff Pol, H. E. (2010). Exploring the brain network: A review on resting-state fMRI functional connectivity. European Neuropsychopharmacology, 20(8), 519-534. https://doi.org/10.1016/j.euroneuro.2010.03.008 
van Harmelen, A.-L. (2016). Friendships and Family Support Reduce Subsequent Depressive Symptoms in At-Risk Adolescents. Retrieved February 9, 2018, from https://www.repository.cam.ac.uk/bitstream/handle/1810/255139/van_Harmelen_ et_al-2016-PLOS_ONE-VoR.pdf?sequence=5

Vyas, A., Pillai, A. G., \& Chattarji, S. (2004). Recovery after chronic stress fails to reverse amygdaloid neuronal hypertrophy and enhanced anxiety-like behavior. Neuroscience, 128(4), 667-673. https://doi.org/10.1016/j.neuroscience.2004.07.013 Warmingham, J. M., Handley, E. D., Rogosch, F. A., Manly, J. T., \& Cicchetti, D. (2018). Identifying maltreatment subgroups with patterns of maltreatment subtype and chronicity: A latent class analysis approach. Child Abuse \& Neglect. https://doi.org/10.1016/j.chiabu.2018.08.013

White, M. G., Bogdan, R., Fisher, P. M., Muñoz, K. E., Williamson, D. E., \& Hariri, A. R. (2012). FKBP5 and emotional neglect interact to predict individual differences in amygdala reactivity. Genes, Brain, and Behavior, 11(7), 869-878.

https://doi.org/10.1111/j.1601-183X.2012.00837.x

White, S. F., Tyler, P., Botkin, M. L., Erway, A. K., Thornton, L. C., Kolli, V., ... Blair, R. J. (2016). Youth with Substance Abuse Histories Exhibit Dysfunctional Representation of Expected Value During a Passive Avoidance Task. Psychiatry Research, 257, 17-24. https://doi.org/10.1016/j.pscychresns.2016.08.010

Widom, C. S., Czaja, S., \& Dutton, M. A. (2014). Child Abuse and Neglect and Intimate Partner Violence Victimization and Perpetration: A Prospective Investigation. Child Abuse \& Neglect, 38(4), 650-663. https://doi.org/10.1016/j.chiabu.2013.11.004 
Will, G.-J., Rutledge, R. B., Moutoussis, M., \& Dolan, R. J. (2017). Neural and computational processes underlying dynamic changes in self-esteem. ELife, 6, e28098. https://doi.org/10.7554/eLife.28098

Wills, J., Hackel, L., \& Bavel, J. J. V. (2018). Shifting prosocial intuitions: Neurocognitive evidence for a value based account of group-based cooperation. PsyArXiv. https://doi.org/10.17605/OSF.IO/U736D

Yarkoni, T. (2009). Big Correlations in Little Studies: Inflated fMRI Correlations Reflect Low Statistical Power-Commentary on Vul et al. (2009). Perspectives on Psychological Science, 4(3), 294-298. https://doi.org/10.1111/j.1745-6924.2009.01127.x 